\title{
Dynamic changes of touch- and laser heat-evoked field potentials of primary somatosensory cortex in awake and pentobarbital-anesthetized rats
}

\author{
Fu-Zen Shaw ${ }^{\mathrm{a}}$, Ruei-Feng Chen ${ }^{\mathrm{b}}$, Chen-Tung Yen ${ }^{\mathrm{b}, *}$ \\ ${ }^{a}$ Institute of Neuroscience, Tzu Chi University, Hualien, Taiwan \\ ${ }^{\mathrm{b}}$ Department of Zoology, National Taiwan University, No. 1, Section 4, Roosevelt Road, Taipei 106, Taiwan
}

Accepted 1 June 2001

\begin{abstract}
In this investigation, changes of mechanical- (MEP) and laser-evoked potentials (LEP) in rat primary somatosensory cortex during the course of pentobarbital (PB) anesthesia were examined. Temporal analysis of changes in the magnitude and latency of MEP and LEP, EEG activity, gross motor behaviors, and the tail flick response following laser stimulation before, during, and after PB administration (50 $\mathrm{mg} / \mathrm{kg}$, i.p.) was performed and correlated in chronically implanted rats. During the wakeful condition, there were two major cortical components each following mechanical stimulation (MEP1 and MEP2, $n=17$ ) and laser stimulation (LEP1 and LEP2, $n=10$ ), respectively. After PB administration, the positive peak in MEP1 was enhanced, and all other components disappeared. These components returned with different time courses. Two hours after PB administration, when the rat had spontaneous movements and flexor reflexes, LEP2 showed reversed polarity. MEP2 returned gradually $3 \mathrm{~h}$ after PB administration when the rat regained its ability to execute coordinated movements. After 4 h, LEP1 began to reappear and LEP2 returned to its negative polarity. We found that PB facilitated A $\beta$ fiber-related cortical evoked potential (MEP1), while differentially inhibited A $\delta$ and C fiber-related components (MEP2, LEP1 and LEP2). Characterization of these anesthesia-induced changes in cortical output may be useful in studying the neural basis of tactile and pain sensations. (C) 2001 Elsevier Science B.V. All rights reserved.
\end{abstract}

Theme: Sensory systems

Topic: Somatosensory cortex

Keywords: Nociception; Pain; C fiber; A $\delta$ fiber; Freely moving rat

\section{Introduction}

Early in the 20th century, Head and Holms [19] reported that 'pure cortical lesions cause no increase or decrease of sensibility to measured painful stimuli'. It was inferred for decades that the cerebral cortex is not involved in human pain processing. Converging clinical and experimental evidence has substantially modified this view over the years. Several cortical areas, such as the primary (SI) and secondary (SII) somatosensory cortices and the anterior cingulate cortex, have been confirmed to process the nociceptive inflow as revealed by data from experimental

\footnotetext{
*Corresponding author. Tel.: +886-2-2363-0231; fax: +886-2-23636837.

E-mail address: ctyen@ccms.ntu.edu.tw (C.-T. Yen).
}

animal and human lesion (for reviews, see Refs. [23,35]), and functional imaging studies (for reviews, see Refs. $[10,20])$. However, the temporal characteristics of nociceptive processing in the cortex have remained largely unknown. Several technologies could be used to address this question. Recording cortical electrical activity elicited by noxious stimuli has a nice temporal resolution in the millisecond range. It will be helpful to understand the temporal properties of nociceptive inflow in the cortex.

Evoked potentials of SI, which are elicited by innocuous and noxious stimulation, have been described in many anesthetized animal studies $[1,4,22]$. In these investigations, a positive and $\mathrm{A} \beta$-fiber related evoked potential (EP) induced by innocuous stimulation is consistently recorded on the surface of the SI $[1,4,12,22,27,30]$. A clear positive and C-fiber related EP responding to noxious laser stimula- 
tion has been demonstrated in halothane-anesthetized rats [22]. It is well known that anesthetics strongly modulate functional properties of cortical neurons $[11,13,15,16,24,31,32,37]$. For instance, major differences in neuronal firing patterns can be observed between the awake and anesthetized condition, especially in long latency evoked responses [11,13,26,29,34,37]. Furthermore, a negative cortical component elicited by innocuous mechanical stimulation exists only in awake monkeys $[11,12,27]$. To avoid these anesthetic effects and to study the underlying cortical responses following innocuous and noxious stimulation, it is necessary to record cortical activity in the conscious animal. On the other hand, detail neurophysiological data can only be obtained in anesthetized condition. Thus it is important to be able to relate responses under anesthetized and conscious conditions.

Recently, the use of short pulse $\mathrm{CO}_{2}$ laser stimulation has been developed for pain research (for reviews, see Refs. [3,6]). The heat stimulus selectively activates nociceptive receptors and generates a pure pain sensation, which is conveyed through both small myelinated A $\delta$ and unmyelinated $\mathrm{C}$ fibers to the cerebral cortex in humans $[7,8]$. Two major cortical evoked components (LEP1 and LEP2), following $\mathrm{CO}_{2}$-laser stimulation of the tail of freely moving rats, are also observed [30]. LEP1 and LEP2 are related to $\mathrm{A} \delta$ and $\mathrm{C}$ afferent fiber activation, respectively [30]. These two specific components elicited by $\mathrm{CO}_{2}$ laser differ greatly from the results obtained from anesthetized animals. For example, LEP1, a component that was observed in conscious rats [30], could not be systematically obtained in halothane-anesthetized rats [22]. In addition, a negative $\mathrm{C}$ fiber-mediated LEP2 component that was found in behaving rats [30] demonstrated positive polarity under halothane anesthesia [22]. In light of druginduced changes evident in cortical evoked activity, it is important to further characterize changes in stimulusevoked components of cortical activity before, during, and after general anesthesia. Correlating temporal alterations of somatosensory electrical potentials in response to innocuous and noxious stimuli with EEG as well as spontaneous/ reflexive behaviors may reveal important functional features integral to the central processing of touch and pain.

\section{Materials and methods}

Adult male Wistar rats (250-300 g) were anesthetized initially with sodium pentobarbital (50 $\mathrm{mg} / \mathrm{kg}$, i.p.). Ketamine was administered as necessary to maintain proper anesthetic depth during surgery. The dorsal surface of the skull was exposed and cleaned. Stainless steel screws (O.D. $=1 \mathrm{~mm}$ ) were driven bilaterally into the skull overlaying the tail representative area of SI. The mean impedance of these electrodes was $7.5 \mathrm{k} \Omega$. Reference electrodes were implanted $2 \mathrm{~mm}$ caudal to lambda. These electrodes did not penetrate the underlying dura. To assure functioning of the electrodes, evoked potentials were elicited by stimulating the tail of the rat using $0.5-\mathrm{ms}$ duration constant current pulses (Grass model 44). Only subjects with identifiable short-latency evoked potentials of positive polarity were used in this study. Dental cement was then applied to fasten the connection module on the surface of the skull. Finally, animals were injected with antibiotics (Combion-S) and housed individually in cages for recovery.

Three weeks later, animals were placed individually in clear acrylic chambers, so that the behavior of the rats could be easily observed. At the top of the chamber, there was a narrow opening through which the head plug/cable assembly was fed to permit unimpeded access to the skull implant. To habituate rats to the experimental apparatus, each animal was placed in the recording environment at least five times ( $2 \mathrm{~h} /$ day) prior to testing. On the day of the recording, a 30-min period was allowed for the rat to become familiar with the chamber. Sodium pentobarbital (50 $\mathrm{mg} / \mathrm{kg}$ ) was administered intra-peritoneally. About 100 mechanical and $\mathrm{CO}_{2}$-laser stimulation trials were applied to the rat's tail hourly, before, during, and after pentobarbital administration. Mechanical-evoked cortical potential (MEP) and LEP were averaged (see below). Surgical and experimental procedures complied with NIH (USA) recommended procedures for animal use and care and were approved by the Experimental Animal Committee of the Tzu Chi University.

A blunt probe attached to a coil was used as an innocuous mechanical stimulator. The probe was displaced by a short current pulse ( $2 \mathrm{~ms}$ ) generated by a Grass stimulator. The stimulator was adjusted to induce light pressure on the skin (15-20 g). The inter-stimulus interval of the mechanical stimulation trial was kept longer than 2 s. In addition, a medical surgical $\mathrm{CO}_{2}$ laser $(10.6 \mu \mathrm{m}$ wavelength, $\mathrm{TEM}_{00}$ mode) was used to excite nociceptors. The unfocused laser beam (3 $\mathrm{mm}$ in diameter) was randomly applied to the skin area at the middle portion of the tail $\left(\sim 100 \mathrm{~mm}^{2}\right)$. The laser intensity was $120 \mathrm{~mJ}$ (12 $\mathrm{W}, 10 \mathrm{~ms})$. The inter-stimulus interval of the laser stimulation trial was longer than $10 \mathrm{~s}$ to minimize sensitization or habituation of nociceptors. The hairs in the middle region of the tail were shaved before the laser experiment. These detailed experimental procedures have been described previously [30,40].

Field potentials from skull electrodes were amplified (4th order band-pass filter: $0.3-1000 \mathrm{~Hz}$, gain=5000×). Each evoked potential was sampled at $4 \mathrm{kHz}$ with a time-locked signal in 1-s segments (4000 data points). Each evoked potential was selected averaged from the responses of about 100 stimulation trials. Data trials were rejected from average if they showed a contaminated movement artifact or large baseline drift. Each selected LEP was filtered with a zero-phase lowpass filter (cut-off frequency $=200 \mathrm{~Hz}$ ) before averaging. Another zero-phase highpass filter (cut-off frequency $=200 \mathrm{~Hz}$ ) was used to 
extract the high frequency signal from original LEP, which correlated with muscle activities induced by tail flick and/or nuchal muscle (orientation) as compared with the behavior record. In addition, the EEG was monitored and stored in order to further evaluate the vigilance state of the rat before, during, and after the pentobarbital anesthesia. An additional lowpass filter (cut-off frequency $=70 \mathrm{~Hz} ; 4$ th order) was used for filtering out high-frequency noise of the amplified field potential. The sampling frequency for the EEG was $250 \mathrm{~Hz}$. EEG data were partitioned into 8-s segments (2000 data points) for calculating the power spectrum. The power spectrum of the EEG was monitored continuously throughout the 5 -h period. In general, barbiturate spikes/spindles were recorded in PB-anesthetized animals [2,33] (Fig. 1). Note that $1 \mathrm{~h}$ after PB administration (Fig. 1, 3rd trace from top), there were many barbiturate spikes and occasionally barbiturate spindles present, indicating a light anesthetic condition at this time [15]. From 1 to $3 \mathrm{~h}$ the shapes of the barbiturate spindles changed progressively from a predominantly negative pattern to a biphasic pattern. The entire data acquisition and analysis program was developed on a LabView (National Instrument, Austin, TX) environment [31].

Magnitudes and latencies of MEP1, MEP2, LEP1, and LEP2, and the ratio and latency of tail flick (TF) elicited by laser stimulation after anesthesia were compared with variations of those values before anesthesia by one-way repeated measures ANOVA. Multiple comparisons among data at different time courses were accomplished using the Student-Newman-Keuls test. After PB administration, the magnitudes of MEP2, LEP1, and LEP2 were sometimes too small to measure quantitatively during the initial phase of anesthesia (see results). During this low-amplitude period, the ceiling scores of the latencies of MEP2, LEP1, and LEP2 were modified and set to 300, 300, and $600 \mathrm{~ms}$, respectively. Data that appear in figures and in the results are expressed as mean \pm S.E.

After the completion of the stimulation and recording experiment, the animal was sacrificed with an overdose of sodium pentobarbital. Special attention was given to

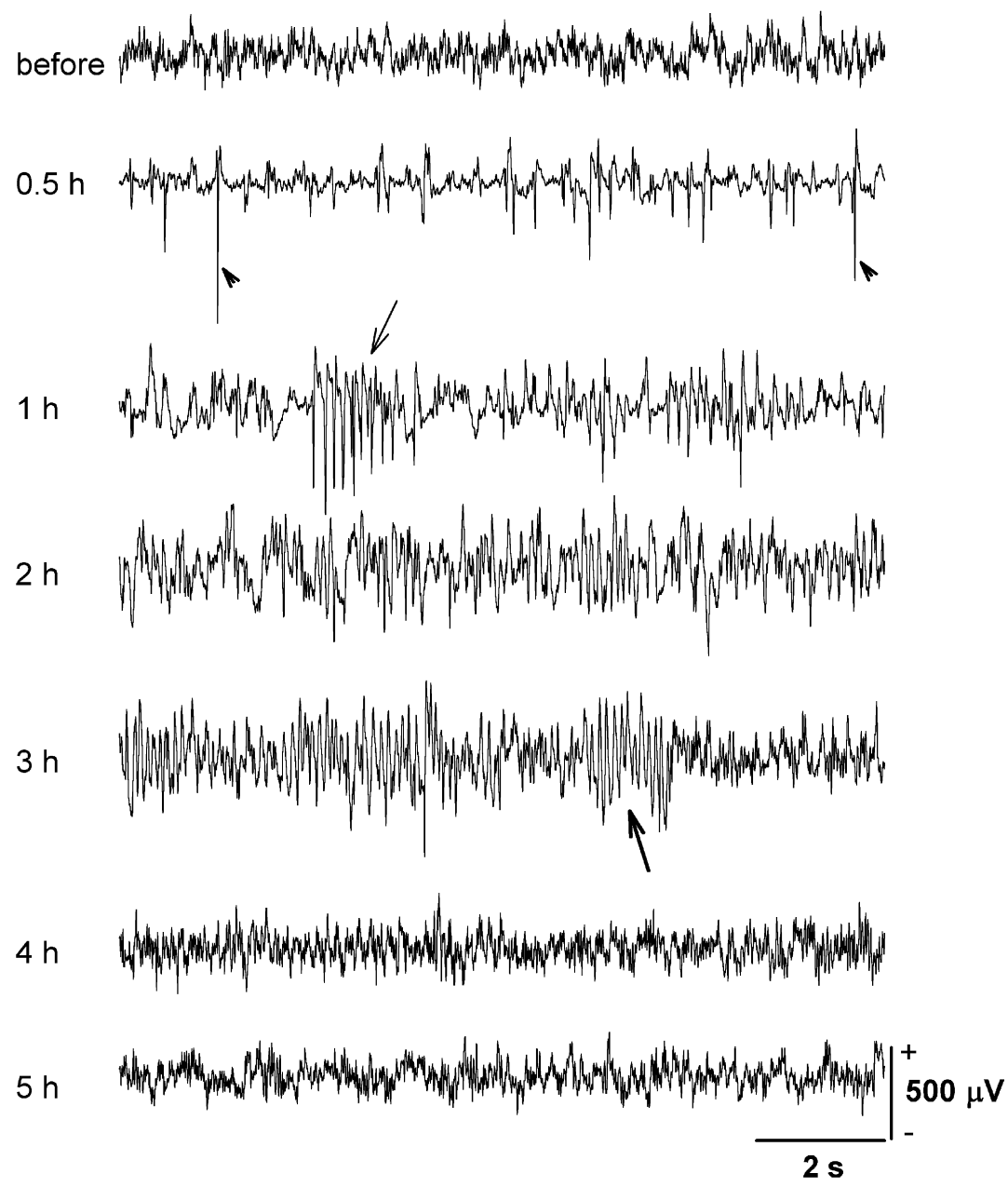

Fig. 1. A representative example of the EEG change in one rat before and after PB administration $(50 \mathrm{mg} / \mathrm{kg}$, i.p.). Barbiturate spikes with a dominant negative polarity were present at 0.5 and $1 \mathrm{~h}$ after PB injection (arrowhead). Barbiturate spindles with a predominantly negative polarity were seen at $1 \mathrm{~h}$ after PB administration (thin arrow). This gradually changed to a biphasic polarity $3 \mathrm{~h}$ after PB injection (thick arrow). In this and the following figures, positivity is oriented upwards. 
ascertain that the stainless steel screws had not penetrated the dura.

\section{Results}

\subsection{Cortical responses to different somatic stimulation before, during, and after anesthesia}

A representative example of the evoked cortical waveforms to innocuous mechanical and noxious $\mathrm{CO}_{2}$ laser stimulation before, during, and after pentobarbital anesthesia is shown in Fig. 2. In the awake condition, the major cortical potentials responding to mechanical stimulation demonstrated a positive peak (MEP1, indicated by

\section{A}

Mechanical

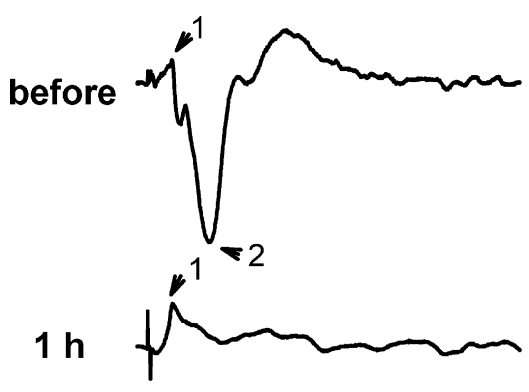

$2 \mathrm{~h}$

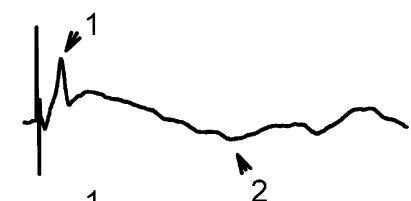

$3 \mathbf{h}$

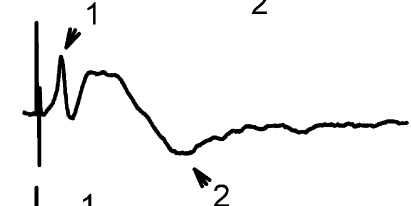

$4 \mathrm{~h}$

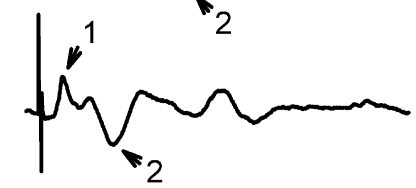

$5 \mathbf{h}$

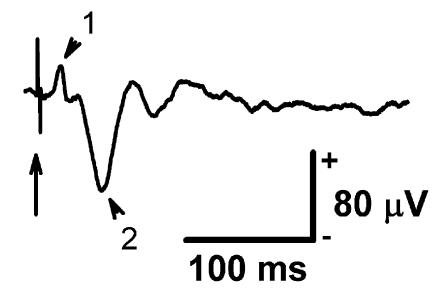

arrowhead 1) followed by a negative peak (MEP2, indicated by arrowhead 2) (Fig. 2A). MEP1 maintained the same polarity throughout the anesthetized condition. The magnitude of MEP1 increased slightly during PB anesthesia. On the other hand, the waveform of MEP2 was dynamically affected by sodium pentobarbital. It disappeared $1 \mathrm{~h}$ after PB administration. A small complex component were seen $2 \mathrm{~h}$ after PB administration. The MEP1 and MEP2 waveform structures began to reappear 2-3 $\mathrm{h}$ after pentobarbital injection. In contrast, following laser stimulation, the cortical evoked potential showed two distinct negative peaks (LEP1 and LEP2) in the awake condition (Fig. 2B). The LEP1 component could not be detected between 1 and $3 \mathrm{~h}$ after PB administration. The initial sign of the LEP1 waveform could be observed $4 \mathrm{~h}$
B $\mathrm{CO}_{2}$-Laser

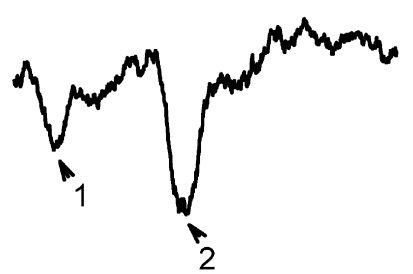

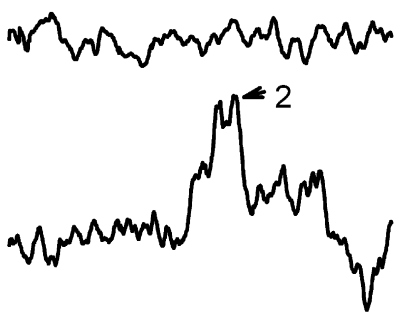

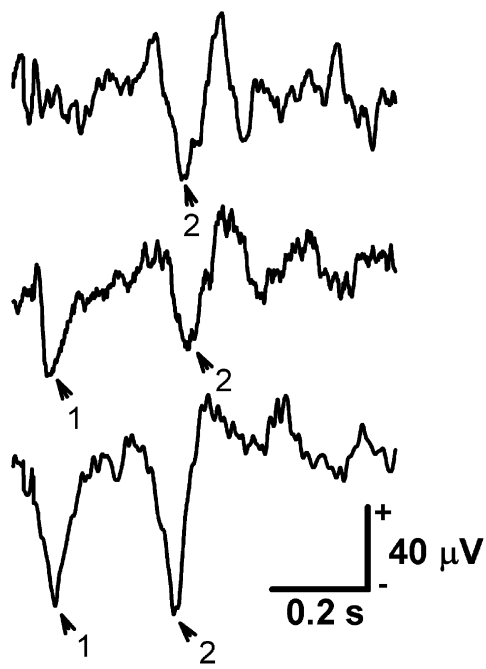

Fig. 2. Examples of MEPs and LEPs recorded over the tail representation of SI. These components were evoked from stimulating the middle portion of the tail before, during, and after PB anesthesia. (A) Mechanical stimulation. The major components of MEP are indicated by arrowheads (1: MEP1; 2: MEP2) in each individual trace. The long arrow indicates the tactile stimulus artifact. (B) Laser heat stimulation. Stimulation pulses (12 W, $10 \mathrm{~ms})$ were given at the beginning of each trace. Arrowheads in each individual trace indicate the major components of LEP. Two distinct negative components (1: LEP1; 2: LEP2) can be seen. 
after PB injection. The LEP2 component, however, disappeared for only an hour. An interesting finding is that the LEP2 component appeared but with a positive polarity $2 \mathrm{~h}$ after PB administration. The polarity of LEP2 then returned to a negative orientation $3 \mathrm{~h}$ after $\mathrm{PB}$ injection. These findings were repeatedly observed in all animals tested.

\subsection{Changes of MEP amplitude and latency before,} during, and after anesthesia

Changes in the magnitude and latency of both MEP1 and MEP2 $(n=17)$ are shown in Fig. 3. During PB anesthesia, rebound oscillation of the mechanical-evoked potential was observed in four rats (open triangle in Fig.
3). These data were not included in the statistical analysis. The magnitude and latency of MEP1 in the control condition $(n=13)$ were $10.5 \pm 1.9 \mu \mathrm{V}$ and $17 \pm 0.3 \mathrm{~ms}$, respectively. After PB administration, MEP1 could be clearly observed in all cases, and the positive polarity of MEP1 was consistently maintained throughout the entire recording period. Changes in the averaged magnitude and averaged latency of MEP1 before and after PB injection indicated a trend $(P<0.001)$ toward an increased magnitude of MEP1 at 1,2 , and $3 \mathrm{~h}$ relative to the wakeful condition (upper part of Fig. 3A). In addition, there was a slight but significant increase in the latency of MEP1 at 1 and $2 \mathrm{~h}$ after $\mathrm{PB}$ administration $(P<0.01)$.

In contrast, the changes in MEP2 after $\mathrm{PB}$ injection followed a markedly different pattern. The peak value and

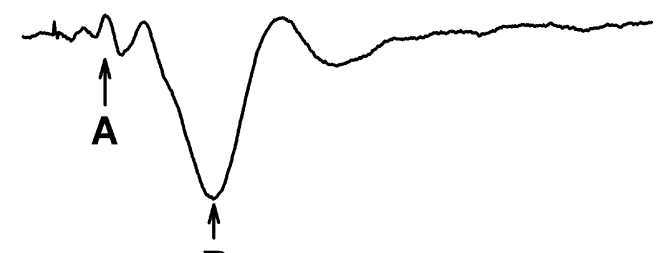

A
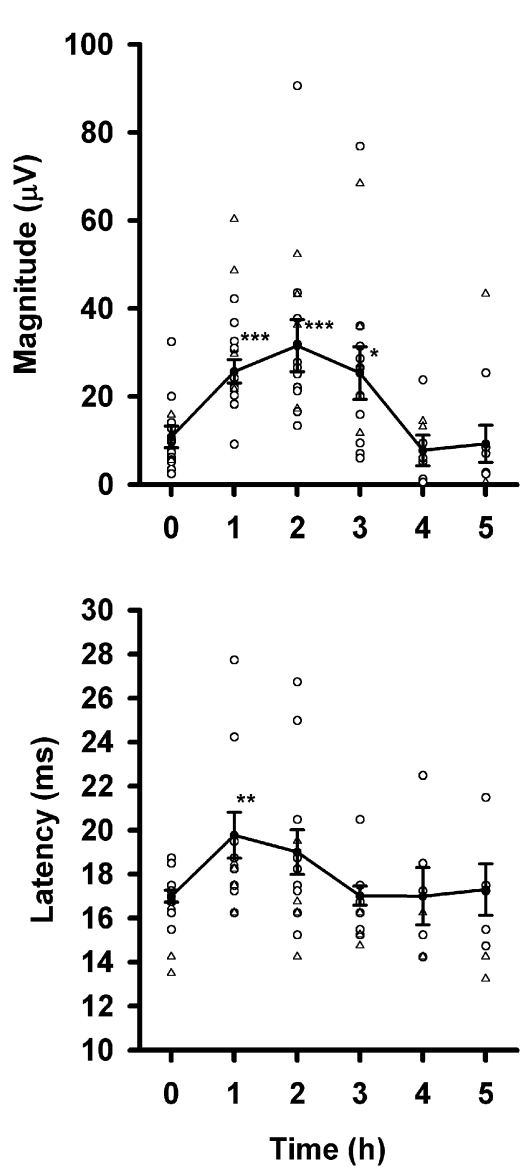

B
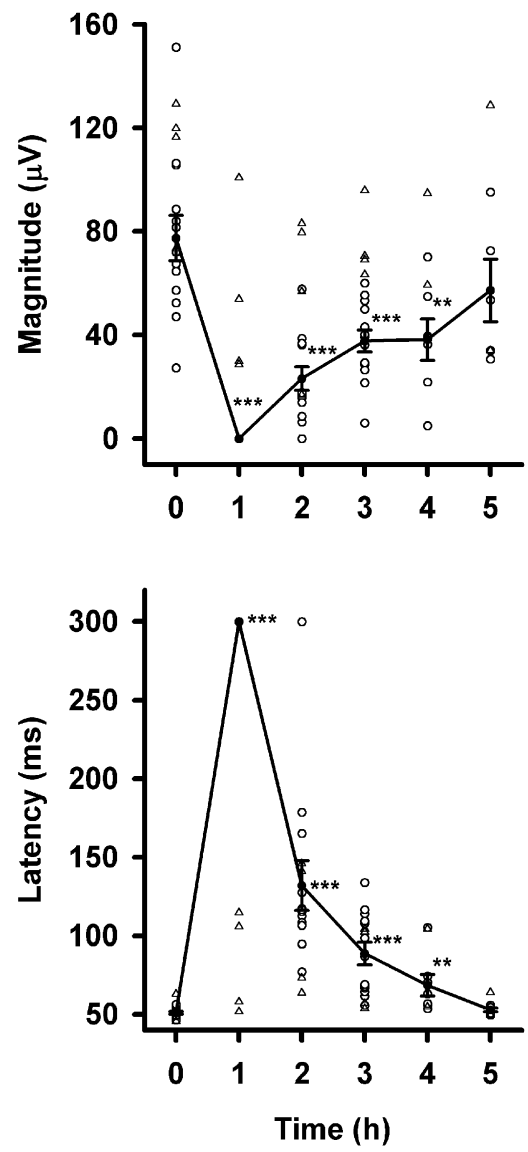

Fig. 3. Changes in the amplitude and latency of MEP1 (A) and MEP2 (B) before and after PB administration. Four rats showed rebound oscillations during PB anesthesia $(\Delta)$, and their data were not included in the statistical analysis. Lines connect averaged values $(\bullet)$ from individual data which are represented by open circles. Error bars are the S.E. of the mean. *** $P<0.001 ; * * P<0.01$; $* P<0.05$. 
the latency of MEP2 before $\mathrm{PB}$ administration were $-77 \pm 9 \mu \mathrm{V}$ and $51 \pm 1 \mathrm{~ms}$, respectively. After PB injection, MEP2 was totally abolished at $1 \mathrm{~h}$. MEP2 began to recover $2 \mathrm{~h}$ after PB injection in all but one rat. Changes in the averaged magnitude and averaged latency for MEP2 before and after $\mathrm{PB}$ injection were significant $(P<0.001)$. As compared to the wakeful condition, differences in the peak value and latency of MEP2 were evident at $1,2,3$, and $4 \mathrm{~h}$ (Fig. 3B). Detailed values of averaged MEP2 magnitude and latency before and after PB injection are depicted in Table 1.

\subsection{Changes of LEP amplitude and latency before, during, and after anesthesia}

Changes in the magnitude and latency of both LEP1 and LEP2 $(n=10)$ are shown in Fig. 4. Relative to MEP, the responses of LEP to $\mathrm{PB}$ anesthesia were more complex. LEP1 was totally abolished at $1 \mathrm{~h}$, and nearly abolished at $2 \mathrm{~h}$ with the exception of one rat after PB injection (Fig. $4 \mathrm{~A})$. Even $3 \mathrm{~h}$ after $\mathrm{PB}$ injection, more than half of the animals (six of ten rats) had no sign of LEP1. LEP1 was restored $4 \mathrm{~h}$ post-PB injection for the entire experimental group. In contrast to the monophasic change of LEP1, the change of LEP2 with PB anesthesia displayed a biphasic pattern (Fig. 4B). One hour after PB injection, the response of LEP2 was almost eliminated, however, three rats showed a positive LEP2 with a small but discernable magnitude. Interestingly, the polarity of LEP2 in all samples was reversed $2 \mathrm{~h}$ post-PB injection and appeared as a positive peak in all animals that were sampled (Fig. 4B). Seven of the ten cases showed LEP2 with negative polarity (except in three rats) $3 \mathrm{~h}$ after $\mathrm{PB}$ injection. The negative LEP2 was totally restored by $4 \mathrm{~h}$ after PB injection.

Three rats, which had a positive LEP2 $3 \mathrm{~h}$ after PB injection (encircled open triangles in the upper panel of Fig. 4B), demonstrated a stronger influence by PB anesthesia. To reduce the possibility that a variance in physio- logical states may have contributed to this small collection of deviant responses, data from these three rats were excluded from the following quantitative analysis. The magnitude and latency of LEP1 $(n=7)$ during the control condition were $-56 \pm 13 \mu \mathrm{V}$ and $83 \pm 5 \mathrm{~ms}$, respectively. Both the magnitude and latency of LEP1 1, 2, and $3 \mathrm{~h}$ post-PB injection differed significantly with the wakeful condition $(P<0.001)$. The magnitude and latency of LEP2 in the wakeful condition were $-100 \pm 27 \mu \mathrm{V}$ and $357 \pm 11$ $\mathrm{ms}$, respectively. Two hours after PB administration, the magnitude and latency of LEP2 were $53 \pm 3 \mu \mathrm{V}$ and $461 \pm 20 \mathrm{~ms}$, respectively. This was a significant $(P<$ $0.001)$ biphasic trend of change of the LEP2 magnitude. In addition, the latency of LEP2 showed a significant trend of increase under PB anesthesia $(P<0.001)$. Detailed values of averaged LEP magnitudes and latencies before and after $\mathrm{PB}$ injection are depicted in Table 1.

\subsection{Changes of tail flick and behavior before, during, and after anesthesia}

Specific behavioral changes were noted before and after $\mathrm{PB}$ injection. One hour after $\mathrm{PB}$ injection, rats showed no sign of spontaneous motor activity. Two hours after PB, the animals showed evidence of reflexive and spontaneous behavior, i.e. chewing, climbing, blinking, and sniffing. However, their movements were not well coordinated at this time. Rats appeared to move and stand up easily without handicap (well-coordinated) $3 \mathrm{~h}$ after PB administration.

The TF reflex elicited by laser stimulation was analyzed with the high frequency component picked up from the cortical stainless steel screw electrode. LEP1 and LEP2 were clearly recorded either with or without TF in wakeful condition (Fig. 5A). The latency of LEP2 lagged to that of the TF, and the magnitude of LEP2 increased when the rat presented TF behavior (Fig. 5A). Interestingly, similar TF reflex were observed while LEP2 polarity reversed during $\mathrm{PB}$ anesthesia, especially $2 \mathrm{~h}$ after PB injection (Fig. 5B).

Table 1

Amplitude and latency of mechanical $(n=13)$ and laser $(n=7)$ evoked potentials and tail flick response to laser stimulation before and after sodium pentobarbital $(50 \mathrm{mg} / \mathrm{kg}$, i.p.)

\begin{tabular}{|c|c|c|c|c|c|c|c|}
\hline & & Before & $1 \mathrm{~h}$ & $2 \mathrm{~h}$ & $3 \mathrm{~h}$ & $4 \mathrm{~h}$ & $5 \mathrm{~h}$ \\
\hline \multirow[t]{2}{*}{ MEP1 } & Peak $(\mu \mathrm{V})$ & $10.5 \pm 1.9$ & $29.3 \pm 3.2 *$ & $33 \pm 4.7 *$ & $28.1 \pm 5.3^{*}$ & $9.3 \pm 2.7$ & $12.9 \pm 6$ \\
\hline & Latency (ms) & $16.6 \pm 0.3$ & $19.2 \pm 0.8^{*}$ & $18.4 \pm 0.8$ & $16.7 \pm 0.4$ & $16.6 \pm 1$ & $16.3 \pm 1.1$ \\
\hline \multirow[t]{2}{*}{ MEP2 } & Peak $(\mu V)$ & $-77 \pm 9$ & $-13 \pm 7^{*}$ & $-32 \pm 6^{*}$ & $-46 \pm 5^{*}$ & $-47 \pm 9^{*}$ & $-64 \pm 14$ \\
\hline & Latency (ms) & $51 \pm 1$ & $249 \pm 23 *$ & $126 \pm 13^{*}$ & $87 \pm 6^{*}$ & $71 \pm 7^{*}$ & $54 \pm 2$ \\
\hline \multirow[t]{2}{*}{ LEP1 } & Peak $(\mu \mathrm{V})$ & $-56 \pm 13$ & $0 \pm 0^{*}$ & $0 \pm 0^{*}$ & $-8.7 \pm 4.5^{*}$ & $-49 \pm 10$ & $-45 \pm 10$ \\
\hline & Latency (ms) & $83 \pm 5$ & $300 \pm 0 *$ & $300 \pm 0 *$ & $213 \pm 41^{*}$ & $84 \pm 3$ & $81 \pm 7$ \\
\hline \multirow[t]{2}{*}{ LEP2 } & Peak $(\mu V)$ & $-100 \pm 27$ & $5 \pm 3 *$ & $53 \pm 3^{*}$ & $-45 \pm 12 *$ & $-87 \pm 34$ & $-86 \pm 24$ \\
\hline & Latency (ms) & $357 \pm 11$ & $565 \pm 22 *$ & $461 \pm 20 *$ & $442 \pm 28^{*}$ & $396 \pm 16$ & $373 \pm 14$ \\
\hline \multirow[t]{2}{*}{ Tail flick } & Proportion (\%) & $57 \pm 6$ & $5 \pm 2 *$ & $37 \pm 5^{*}$ & $47 \pm 3$ & $46 \pm 3$ & $47 \pm 4$ \\
\hline & Latency (ms) & $318 \pm 12$ & $600 \pm 0 *$ & $393 \pm 9 *$ & $372 \pm 13 *$ & $353 \pm 14$ & $325 \pm 14$ \\
\hline
\end{tabular}

MEP1: mechanical positive peak; MEP2: mechanical negative peak; LEP1: 1st negative laser-evoked peak; LEP2: 2nd negative laser-evoked peak. If the peak could not be identified, the ceiling latencies of MEP2, LEP1, and LEP2 were set to 300, 300, and 600 ms, respectively. Data are mean \pm S.E.

* Indicates value significantly different from control (before) value. 

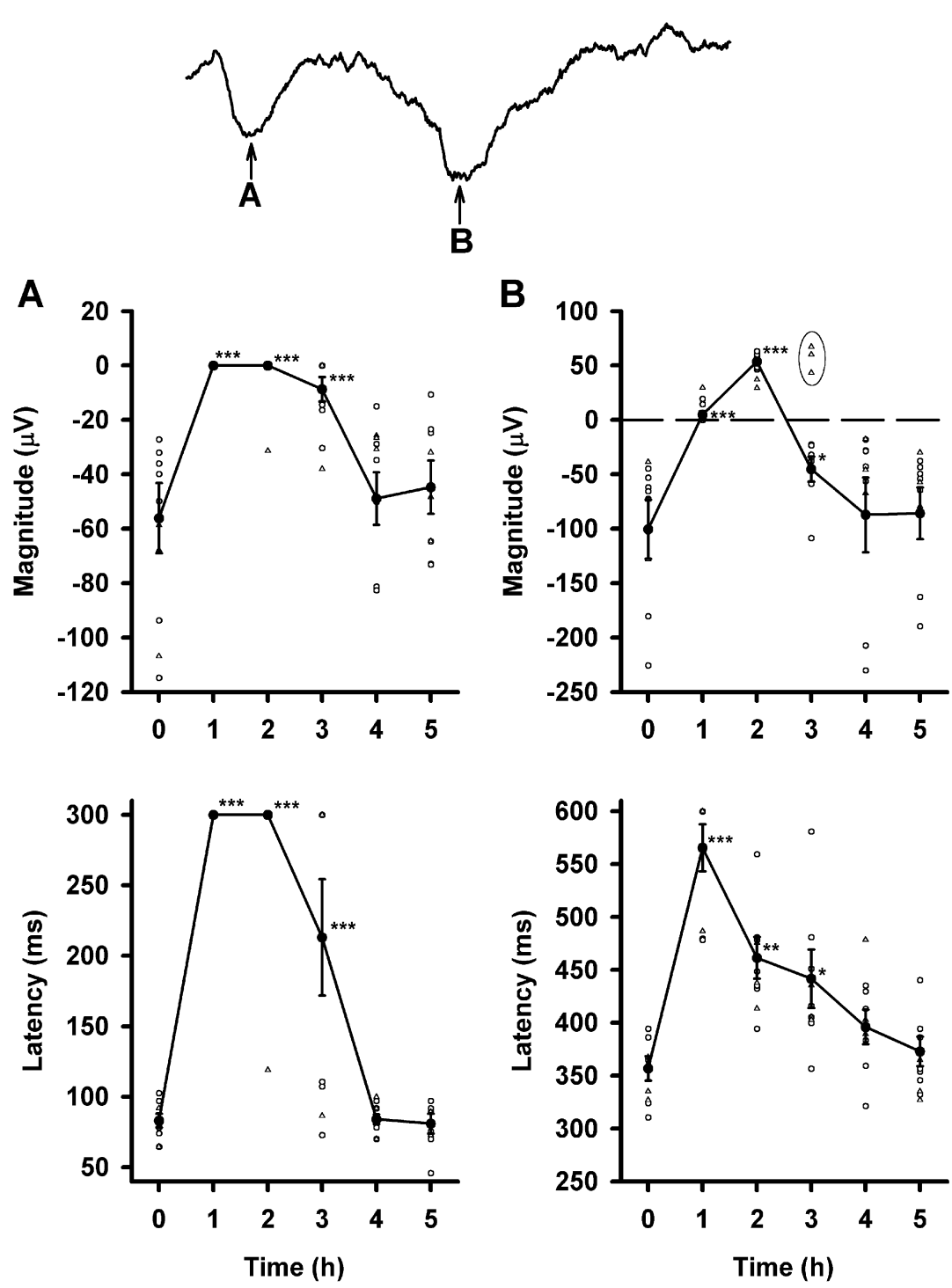

Fig. 4. Changes in the amplitude and latency of LEP1 (A) and LEP2 (B) before and after PB administration. LEP1 was totally abolished $1 \mathrm{~h}$ after PB administration, and then gradually returned. The response of LEP1 shows a monophasic change. LEP2 was reversed and peaked in a positive direction $2 \mathrm{~h}$ after PB administration in all cases. LEP2 gradually re-established a negative peak. The response of LEP2 shows a biphasic change. Three rats showed a positive polarity $3 \mathrm{~h}$ after PB administration $(\Delta)$. Their data were not included in the statistical analysis. Lines connect averaged values $(-)$ from the individual data shown with open circles. Error bars are the S.E. of the mean. $* * * P<0.001 ; * * P<0.01$; $* P<0.05$.

Over $50 \%(57 \pm 6 \%)$ of laser stimulation was accompanied by a TF response before PB injection. A dramatic decrease in $\mathrm{TF}$ was observed $1 \mathrm{~h}(5 \pm 2 \%)$ after $\mathrm{PB}$ injection $(P<0.001)$. Ratio of TF response restored to normal $3 \mathrm{~h}$ after PB injection (Table 1). We selected 20 high-frequency muscle responses randomly for the calculation of averaged latency to laser stimulation before, during and after PB administration. The latency of TF reflex during the wakeful condition by laser stimulation was $318 \pm 12$ $\mathrm{ms}$. TF latency increased significantly 1,2 , and $3 \mathrm{~h}$ after PB injection. It returned to comparable control values $4 \mathrm{~h}$ after. Detailed values of averaged proportion and latency of TF to laser stimulation before and after PB administration are depicted in Table 1. Changes in the polarity of
MEP and LEP as well as in behaviors and EEG before and after PB treatment are summarized in Table 2.

\section{Discussion}

We describe herein dynamic and differential changes of mechanical and laser heat evoked potentials recorded over the cortical area of the SI representing the tail following pentobarbital administration. These components returned with different time courses, and interesting correlations were found with respect to the behavior of the rat (Table 2). 
A

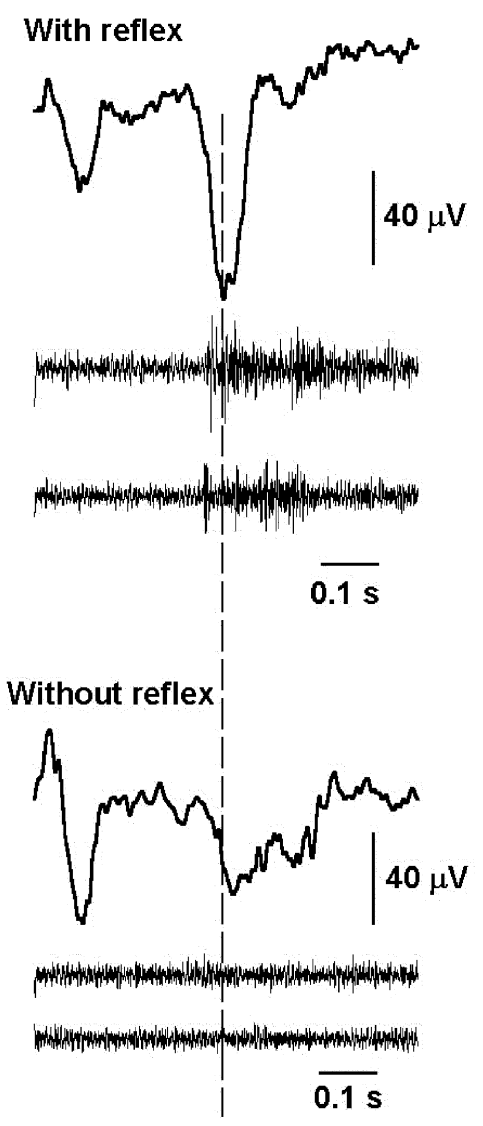

B

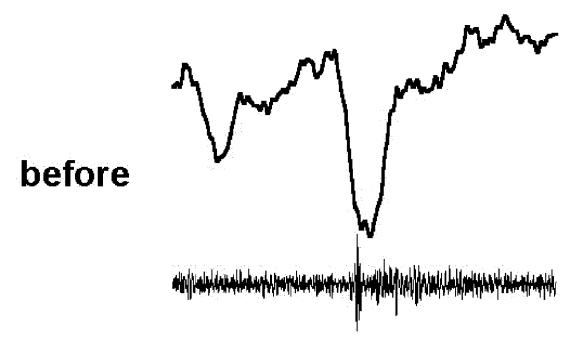

$2 \mathrm{~h}$

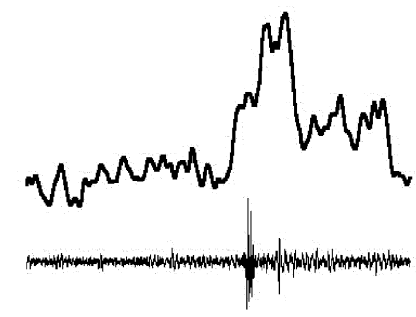

$5 \mathrm{~h}$

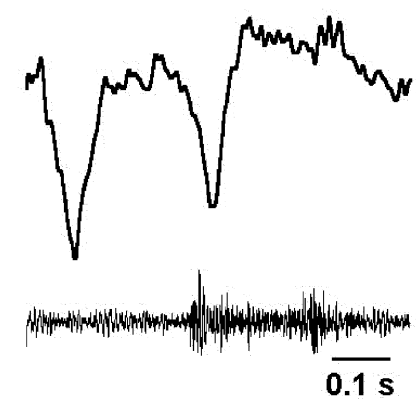

Fig. 5. Laser heat-evoked potential and reflexive muscle activity. (A) LEP and muscle activity with/without tail flick (TF) in the wakeful condition. Note that LEP1 and LEP2 are consistently recorded, with or without TF response. The average number of LEP in upper and lower panels is about 50 and 30 , respectively. The muscle activity traces are randomly selected single trials. (B) Averaged LEP and reflexive muscle activities before and after PB anesthesia. Note LEP2 latency closely correlated TF latency.

Table 2

Comparison of MEP, LEP, spontaneous behavior, tail flick response, and EEG before and after sodium pentobarbital injection $(50 \mathrm{mg} / \mathrm{kg}$, i.p.)

\begin{tabular}{lllllll}
\hline & Before & $1 \mathrm{~h}$ & $2 \mathrm{~h}$ & $3 \mathrm{~h}$ & $4 \mathrm{~h}$ & $5 \mathrm{~h}$ \\
\hline MEP1 & $\mathrm{P}$ & $\mathrm{P}$ & $\mathrm{P}$ & $\mathrm{P}$ & $\mathrm{P}$ & $\mathrm{P}$ \\
MEP2 & $\mathrm{N}$ & $\mathrm{NP}$ & $\mathrm{N}$ & $\mathrm{N}$ & $\mathrm{N}$ & $\mathrm{N}$ \\
LEP1 & $\mathrm{N}$ & $\mathrm{NP}$ & $\mathrm{NP}$ & $\mathrm{NP}$ & $\mathrm{N}$ & $\mathrm{N}$ \\
LEP2 & $\mathrm{N}$ & $\mathrm{NP}$ & $\mathrm{P}$ & $\mathrm{N}$ & $\mathrm{N}$ & $\mathrm{N}$ \\
Behavior & ++ & $\mathrm{A}$ & + & ++ & ++ & ++ \\
Tail flick & Yes & $\mathrm{NP}$ & $\mathrm{SS}$ & $\mathrm{SS}$ & Yes & Yes \\
EEG & Normal & $\mathrm{BS}$ & $\mathrm{BD}$ & $\mathrm{BD}$ & Normal & Normal \\
\hline
\end{tabular}

MEP1: mechanical positive peak; MEP2: mechanical negative peak; LEP1: 1st negative laser-evoked peak; LEP2: 2nd negative laser-evoked peak; P: positive polarity; N: negative polarity; NP: not present; A: anesthetized; + : movement not well coordinated; ++ : well-coordinated movement and behavior (move and stand up easily without handicap); SS: significantly suppressed comparing with the awake condition; BS: barbiturate spikes; BD: barbiturate spindles.

\subsection{Mechanical evoked potential}

In our previous investigation [30], we suggested that MEP1 is mediated by A $\beta$ afferent fibers, MEP2 and LEP1 by $A \delta$ afferent fibers, and LEP2 by $\mathrm{C}$ fibers. Our present results demonstrate that the A $\beta$-fiber-related MEP1 component maintained a positive polarity, and that the magnitude of MEP1 was enhanced during light anesthesia. These results are consistent with those published in other studies $[1,4,15,16,30]$. The exact mechanism by which barbiturates potentiate MEP1 is unclear. It is well known that barbiturates positively modulate the effects of the inhibitory transmitter, GABA [41], which is abundant within the cerebral cortex. This will attenuate the spontaneous activity of cortical neurons; thereby enhance the signal to noise ratio of MEP1. Alternatively, barbiturates may block the ascending inhibitory components from the reticular formation and the end result is an enhanced evoked response during light $\mathrm{PB}$ anesthesia condition $[15,16]$. 
In this study, we observed that MEP2 disappeared as rats became anesthetized ( $1 \mathrm{~h}$ after PB injection). MEP2 returned with a time course similar to the outward behavior of the rat, such that at $2 \mathrm{~h}$ when the rat moved about staggeringly, traces of MEP2 was observed; and at $3 \mathrm{~h}$ when the rat regained its pose, MEP2 could be consistently recorded. Several reports also indicate that a long latency negative component (comparable to the MEP2 here) elicited by innocuous mechanical stimulation can be clearly recorded in wakeful humans [39], monkeys [12,27], and rats [30]. In addition, this negative component is abolished/attenuated during sleep in humans [39] and monkeys [12]. These observations are consistent with the hypothesis that MEP2 is a behaviorally related cortical component [11].

\subsection{Laser heat evoked potential}

Our recent study [30] has shown that MEP2 and LEP1 have similar waveforms with negative polarity that are probably mediated by A $\delta$ afferent fibers. However, there are several interesting differences. First, the latencies of MEP2 and LEP1 during the wakeful condition differ, being $51 \pm 1$ and $83 \pm 5 \mathrm{~ms}$, respectively. Thermal conductive time from surface to receptor level of the skin by laser stimuli has been estimated to be $30-60 \mathrm{~ms}[7,17]$. This might account for the latency difference. Another possibility is that MEP2 represents polysynaptic activation of $\mathrm{A} \beta$ fiber from the mid-tail, thereby arriving the SI earlier than LEP1. Second, over the cortical surface, the MEP2 component is concentrated in the SI [30] and is primarily associated with superficial neuronal activity $[12,27,30]$. In contrast, LEP1 is small and confined to the SI in its initial positive phase, but becomes more widespread within the sensory-motor region during the larger negative phase [30]. Also, LEP1 is associated with multi-layer cortical activity [30]. The multi-layered distribution of intra-cortical neuronal activity as well as the more diffusely distributed topographic mapping of LEP1 resembles activity observed following tooth-pulp stimulation, which primarily activates nociceptive A $\delta$ afferent inputs [14,28]. Lastly, we further demonstrate in this study that both MEP2 and LEP1 are profoundly affected by PB anesthesia but with different time courses of recovery. Among these cortical EPs, LEP1 is most sensitive to anesthesia. $2 \mathrm{~h}$ after PB injection, LEP1 could be detected in one of seven rats. Even $3 \mathrm{~h}$ after, LEP1 was only barely detectable in most of the rats tested (Fig. 4A). In contrast, MEP2 had begun to return an hour previously (Fig. 2A). The return time course of the LEP1 paralleled that of the tail flick response more closely than MEP2. Based on these observations, we postulate that LEP1 has more functional characteristics than does MEP2. Like MEP2, LEP1 may be related to consciousness, but it may play a further role in processing the nociceptive inflow.

An interesting polarity reversal of the C-fiber related
LEP2 had been reported previously [30,31]. We present in this report a quantitative analysis of this phenomenon (Fig. 4B) and its relationship with the reflexive muscle activities (Fig. 5). In contrast to the very sensitive LEP1, LEP2 can be recorded under light anesthesia [22]. Anesthesia, however, causes a longer-lasting change of the LEP2 than previously known. Thus, LEP2 showed a reversed polarity under light anesthesia ( $1 \mathrm{~h}$ after), when the rat was able to walk about ( $2 \mathrm{~h}$ after), and also in a few rats with well-coordinated behavior $3 \mathrm{~h}$ after (encircled three rats in Fig. 4B). It was $4 \mathrm{~h}$ after a single PB injection that the LEP2 returned to its control level.

Three hours after a single dose of $\mathrm{PB}$, there remained in the central nervous system significant drug effect even the rat showed no apparent behavior handicap. This longer than expected PB effect has an immediate practical consequence. Many behavioral and neurophysiological studies use chronically instrumented rats. If the tettered recording line should be connected with the aid of a premedication, then there should be an ample waiting period for the drug effect (especially anesthetics) to wear off completely. Results of this report indicate that an hour more than the return of the apparent normal gaits and locomotion will be wise. Also, there seems to be a curious dissociation of behavior and central nociceptive processing such that at 3 $\mathrm{h}$ after $\mathrm{PB}$ injection, the rats had regained coordinated behavior but the EPs and tail flick response were still abnormal. The remaining central drug effect is evident only with the aid of EP and muscle activity monitoring. Therefore, it seems likely that if more refined motor tasks were used, some handicaps might have been detected.

In other reports related to C-fiber-related cortical responses, Kalliomaki et al. [22] also described a positive polarity C-fiber component evoked by laser heat stimulation in halothane anesthetized rats. This reversal of polarity has also been observed by Shyu et al. [34]. This group found that the superficial cortical evoked potential responded to central lateral nucleus stimulation changes from negative to positive polarity after PB administration. Thus, it seems that during the wakeful condition, $\mathrm{C}$ fiber activation evokes a widespread, superficial layer dominant component in the cerebral cortex. This superficial cortical component is strongly affected by anesthetics [11,37,38], and is thereby differentially suppressed under light anesthesia. LEP2 changes to positive because at this point, neurons in the deeper cortical layers are still activated [22].

Short-pulse noxious $\mathrm{CO}_{2}$-laser radiation has been demonstrated to be capable of activating both $\mathrm{A} \delta$ and $\mathrm{C}$ nociceptive afferent fibers in rats $[17,18]$ and humans [7]. Both $\mathrm{A} \delta$ and $\mathrm{C}$ fiber-related cortical components can be elicited by laser stimulation in awake rats. Cortical responses to laser stimulation in conscious humans differ from those recorded in awake rats. Although $\mathrm{CO}_{2}$ laser stimulus is able to activate two different kinds of nociceptive peripheral fibers, only the A fiber-related component is observed in normal human studies [7,8,21]. The $\mathrm{C}$ 
fiber-related cortical component can be demonstrated in healthy subjects with special treatment, such as pressure block process [9], minute localized skin stimulation [5], specially designed temperature stimulation [25], or in patients with neurological disorders [6,36]. Even under these situations, only one cortical response, either $\mathrm{A} \delta$ or $\mathrm{C}$, could be evoked by laser stimulation in humans. The mechanism for these differential laser-evoked cortical responses between humans and rats is unclear. It may originate from a dissimilar modulation in spino-thalamocortical pathways. The rat may provide a model system to study the cortical function from both $\mathrm{A} \delta$ and $\mathrm{C}$ nociceptive inputs at the same time. The dissociation of MEPs and LEPs during anesthesia indicates that separate central pathways might be subserving the processing of mechanical $\mathrm{A} \delta$, thermal heat $\mathrm{A} \delta$, and thermal heat $\mathrm{C}$ inputs in the rat.

\subsection{Tail flick and LEP}

The high frequency component $(200-1000 \mathrm{~Hz})$ of the electrical signal recorded from the cortical electrode (a stainless steel screw $1 \mathrm{~mm}$ in diameter) was used to represent the jerky reflexive muscle activities evoked by laser irradiation. From our experience, this is a good index of the tail flick of the rat. Because with the moderate laser intensity used $(10 \mathrm{~ms}, 12 \mathrm{~W})$, tail flicks were elicited about $50 \%$ of the time (Table 1). All rats had been tamed to the procedure. Under this condition, tail flick was usually the only observable movement. The large high frequency signals recorded (such as illustrated in Fig. 5) were unlikely to be originated inside the cortex because in no case was the dura penetrated (see Section 2).

Based on the assumption that these high frequency spikes were originated from the tail flick muscles, another question arises. Since LEP2 latency, amplitude and duration related closely to tail flick responses (Fig. 5, Table 1), could it be that LEP2 might have been a far-field potential originated from distant muscles? We think this is not the case and LEP2 has a near-field cortical origin based on the following evidences. First, with or without tail flick, LEP2 could be recorded (Fig. 5A). Second, if LEP2 is indeed a far-field potential, it should not change its polarity under anesthesia since the same tail was flicking. Third, in a previous report [30], we made multi-channel intra-cortical recording in several cortical laminae. Clear neuronal responses were observed at LEP2 latency after laser heat stimulation of the mid-tail. Similar experiment was performed by Kalliomaki et al. [22]. They reached the same conclusion that C-fiber related LEP was cortical in origin.

Tail flick response, including its latency and amplitude, has been used routinely as an index of pain sensitivity. Considering pain sensitivity changes dynamically, it is reasonable that LEP2 latency and amplitude should correlate with those of the tail flick response. When further details are known, it may be possible to use LEPs as indices of central pain processing.

In this study, we used two specific stimuli to evaluate functional changes in brain activity influenced by PB. The first was an innocuous mechanical stimulus and the second was a noxious thermal stimulus. We described dynamic changes of tactile- and thermal nociceptive-evoked potentials in rat cortex during barbiturate anesthesia. Subtle correlations exist between these changes and reflexive and spontaneous behaviors of the rat. We conclude that PB strongly modifies various components of MEP and LEP. Changes in these components may provide a good model system for the study of the neural basis of tactile and pain sensations.

\section{Acknowledgements}

We thank Professor R.C.S. Lin and Dr K.M. Simpson, University of Mississippi Medical Center, USA, for their helpful comments. The present study was supported by a Cognitive Center grant from the Academia Sinica and grants NSC89-2314-B320-008-M08 to FZS and NSC892311-B002-039 to CTY from the National Science Council of Republic of China.

\section{References}

[1] T. Allison, A.L. Hume, A comparative analysis of short-latency somatosensory evoked potentials in man, monkey, cat, and rat, Exp. Neurol. 72 (1981) 592-611.

[2] P. Andersen, S.A. Andersson, Physiological Basis of the Alpha Rhythm, Appleton-Century-Crofts, New York, 1968.

[3] L. Arendt-Nielsen, Characteristics, detection, and modulation of laser-evoked vertex potentials, Acta Anaesthesiol. Scand. 38 (Suppl. 98) (1994) 1-44.

[4] J.C. Arezzo, H.G. Vaughan Jr., A.D. Legatt, Topography and intracranial source of somatosensory evoked potentials in the monkey. II. Cortical components, Electroencephalogr. Clin. Neurophysiol. 28 (1981) 1-18.

[5] D. Bragard, A.C.N. Chen, L. Plaghki, Direct isolation of ultra-late (C-fibre) evoked brain potentials by $\mathrm{CO}_{2}$ laser stimulation of tiny cutaneous surface areas in man, Neurosci. Lett. 209 (1996) 81-84.

[6] B. Bromm, J. Lorenz, Neurophysiological evaluation of pain, Electroencephalogr. Clin. Neurophysiol. 107 (1998) 227-253.

[7] B. Bromm, R.-D. Treede, Nerve fibre discharges, cerebral potentials and sensations induced by $\mathrm{CO}_{2}$ laser stimulation, Hum. Neurobiol. 3 (1984) 33-40.

[8] B. Bromm, R.-D. Treede, Pain related cerebral potentials: late and ultralate components, Int. J. Neurosci. 33 (1987) 15-23.

[9] B. Bromm, H. Neitzel, A. Tecklenburg, R.-D. Treede, Evoked cerebral potential correlates of $\mathrm{C}$ fibre activity in man, Neurosci. Lett. 43 (1983) 109-114.

[10] K.L. Casey, Forebrain mechanisms of nociception and pain: analysis through imaging, Proc. Natl. Acad. Sci. USA 96 (1999) 7668-7674.

[11] L.J. Cauller, Layer I of primary sensory neocortex: where top-down converges upon bottom-up, Behav. Brain Res. 71 (1995) 163-170.

[12] L.J. Cauller, A.T. Kulics, A comparison of awake and sleeping cortical states by analysis of the somatosensory-evoked response of 
postcentral area 1 in rhesus monkey, Exp. Brain Res. 72 (1988) $584-592$.

[13] J.K. Chapin, D.J. Waterhouse, D.J. Woodward, Differences in cutaneous sensory response properties of single somatosensory cortical neurons in awake and halothane anesthetized rats, Brain Res. Bull. 6 (1981) 63-70.

[14] E.H. Chudler, W.K. Dong, Y. Kawakami, Tooth pulp-evoked potentials in the monkey: cortical surface and intracortical distribution, Pain 22 (1985) 221-233.

[15] D.L. Clark, B.S. Rosner, Neurophysiological effects of general anesthetics. I. The electroencephalogram and sensory evoked responses in man, Anesthesiology 38 (1973) 564-582.

[16] N. Dafny, Neurophysiological approach as a tool to study the effects of drugs on the central nervous system: dose effect of pentobarbital, Exp. Neurol. 59 (1978) 263-274.

[17] P.J. Danneman, J.A. Kiritsy-Roy, T.J. Morrow, K.L. Casey, Central delay of the laser-activated rat tail-flick reflex, Pain 58 (1994) $39-44$.

[18] M. Devor, A. Carmon, R. Frostig, Primary afferent and spinal sensory neurons that respond to brief pulses of intense infrared laser radiation: a preliminary survey in rats, Exp. Neurol. 76 (1982) 483-494.

[19] H. Head, G. Holms, Sensory disturbances from cerebral lesions, Brain 34 (1911) 102-254.

[20] M. Ingvar, J.-C. Hsieh, The image of pain, in: R.D. Wall, R. Welzack (Eds.), Textbook of Pain, Churchill Livingstone, New York, 1999, pp. 215-233.

[21] R. Kakigi, C. Endo, R. Neshige, Y. Kuroda, H. Shibasaki, Estimation of conduction velocity of A $\delta$ fibers in humans, Muscle Nerve 14 (1991) 1193-1196

[22] J. Kalliomaki, H.-R. Weng, H.-J. Nilsson, J. Schouenborg, Nociceptive $\mathrm{C}$ fiber input to the primary somatosensory cortex (SI). A field potential study in the rat, Brain Res. 622 (1993) 262-270.

[23] D.R. Kenshalo, W.D. Willis, The role of cerebral cortex in pain system, in: A. Peter, E.G. Jones (Eds.), Cerebral Cortex: Normal and Altered States of Function, Vol. 9, Plenum Press, New York, 1991, pp. $153-212$.

[24] E. Kochs, R.-D. Treede, J. Schulte am Esch, B. Bromm, Modulation of pain-related somatosensory evoked potentials by general anesthesia, Anesth. Analg. 71 (1990) 225-230.

[25] W. Magerl, Z. Ali, J. Ellrich, R.A. Meyer, R.-D. Treede, C- and A $\delta$-fiber components of heat-evoked cerebral potentials in healthy human subjects, Pain 82 (1999) 127-137.

[26] J. Montagne-Clavel, J.-L. Oliveras, G. Martin, Single-unit recordings at dorsal raphe nucleus in the awake-anesthetized rat: sponta- neous activity and response to cutaneous innocuous and noxious stimulations, Pain 60 (1995) 303-310.

[27] N.N. Peterson, C.E. Schroeder, J.C. Arezzo, Neural generators of early cortical somatosensory evoked potentials in the awake monkey, Electroencephalogr. Clin. Neurophysiol. 96 (1995) 248-260.

[28] A. Roos, B. Rydenhag, S.A. Andersson, Cortical responses evoked by tooth pulp stimulation in the cat. Surface and intracortical responses, Pain 14 (1982) 247-265.

[29] P.S. Sebel, C.P. Heneghan, D.A. Ingram, Evoked responses - a neurophysiological indicator of depth of anesthesia?, Br. J. Anaesth. 57 (1985) 841-842.

[30] F.-Z. Shaw, R.-F. Chen, H.-W. Tsao, C.-T. Yen, Comparison of touch- and laser heat-evoked cortical field potentials in conscious rats, Brain Res. 824 (1999) 183-196.

[31] F.-Z. Shaw, R.-F. Chen, H.-W. Tsao, C.-T. Yen, A multichannel system for recording and analysis of cortical field potentials in freely moving rats, J. Neurosci. Methods 88 (1999) 33-43.

[32] Y. Shigenaga, R. Inoki, Effects of morphine and barbiturate on the SI and SII potentials evoked by tooth pulp stimulation of rats, Eur. J. Pharmacol. 36 (1976) 347-353.

[33] M. Steriade, E.G. Jones, D.A. McCormick, Thalamus, Organization and Function, Vol. 1, Elsevier, New York, 1997.

[34] B.C. Shyu, B. Olausson, B. Rydenhag, Field potential analysis of the cortical projection of the central lateral nucleus in the cat, Acta Physiol. Scand. 137 (1989) 503-512.

[35] R.-D. Treede, D.R. Kenshalo, R.H. Gracely, A.K.P. Jones, The cortical representation of pain, Pain 79 (1999) 105-111.

[36] R.-D. Treede, J. Lankers, A. Frieling, W.H. Zangemeister, K. Kunze, B. Bromm, Cerebral potentials evoked by painful laser stimuli in patients with syringomyelia, Brain 114 (1991) 1595-1607.

[37] B.A. Vogt, The role of layer I in cortical function, in: A. Peter, E.G. Jones (Eds.), Cerebral Cortex, Normal and Alerted States of Function, Vol. 9, Plenum Press, New York, 1991, pp. 49-80.

[38] W.D. Willis, The Pain System, Karger, New York, 1985.

[39] T. Yamada, S. Kameyama, Y. Fuchigami, Y. Nakazumi, Q.S. Dickins, J. Kimura, Changes of short latency latency somatosensory evoked potential in sleep, Electroencephalogr. Clin. Neurophysiol. 70 (1988) 126-136.

[40] C.-T. Yen, C.-H. Huang, S.-E. Fu, Surface temperature change, cortical evoked potential and pain behavior elicited by $\mathrm{CO}_{2}$ lasers, Chin. J. Physiol. 37 (1994) 193-199.

[41] S.A. Zimmerman, M.V. Jones, N.L. Harrison, Potentiation of $\gamma$ aminobutyric acid $_{A}$ receptor $\mathrm{Cl}^{-}$current correlates with in vivo anesthetic potency, J. Pharmacol. Exp. Ther. 270 (1994) 987-991. 